\title{
Spheromak Reactor: Physics Opportunities and Issues
}

\author{
E. Bickford Hooper \\ Lawrence Livermore National Laboratory \\ T. Kenneth Fowler \\ University of California at Berkeley
}

\section{JuL 01 tes \\ OSI}

This paper was prepared for submittal to 1996 ANS Annual Meeting and Embedded Topical Meetings

Reno, Nevada

June 16-20, 1996

June 20, 1996
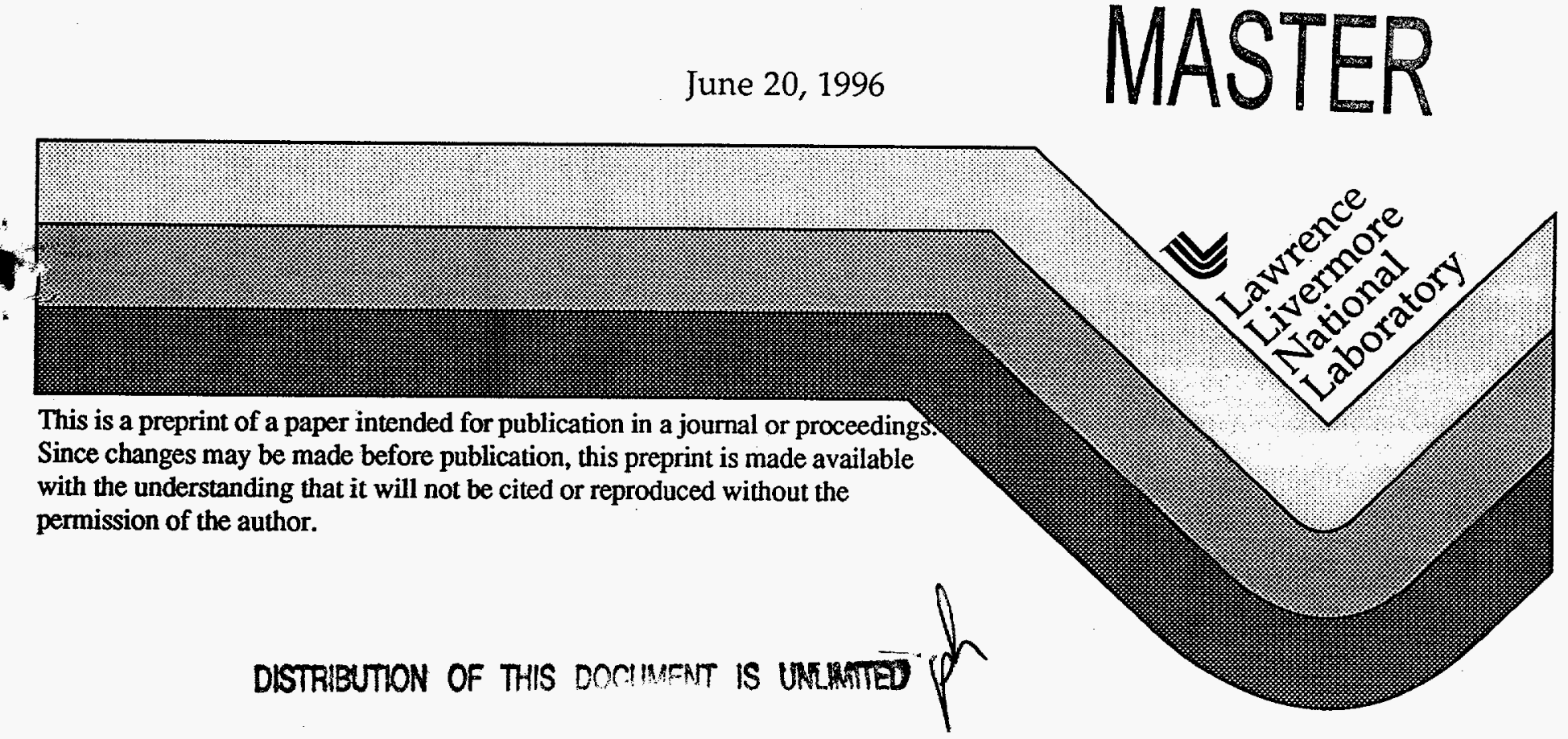


\section{DISCLAIMER}

This document was prepared as an account of work sponsored by an agency of the United States Govemment. Neither the United States Government nor the University of California nor any of their employees, makes any warranty, express or implied, or assumes any legal liability or responsibility for the accuracy, completeness, or usefulness of any information, apparatus, product, or process disclosed, or represents that is use would not infringe privately owned rights. Reference herein to any specific commercial product, process, or service by trade name, trademark, manufacturer, or otherwise, does not necessarily constitute or imply its endorsement, recommendation, or favoring by the United States Government or the University of California. The views and opinions of authors expressed herein do not necessarily state or reflect those of the United States Government or the University of Califomia, and shall not be used for advertising or product endorsement purposes. 


\section{DISCLAIMER}

Portions of this document may be illegible in electronic image products. Images are produced from the best available original document. 


\section{SPHEROMAK REACTOR: PHYSICS OPPORTUNITIES AND ISSUES}

\author{
E. Bickford Hooper \\ Lawrence Livermore National Laboratory \\ P.O. Box 808 \\ Livermore, CA 94551 \\ (510)423-1409
}

\author{
T. Kenneth Fowler \\ Department of Nuclear Engineering \\ University of California at Berkeley \\ Berkeley, CA 94720 \\ (510)642-7071
}

\begin{abstract}
The spheromak is a magnetic confinement device with a more attractive fusion reactor potential than the leading geometry, the tokamak. This results in large part from the absence of a toroidal field coil and other structures linking the plasma along the geometric axis. However, because of the lack of a strong external magnetic field, the physics is more complex so that considerable research is required to learn how to achieve the reactor potential. Several critical physics issues are considered here, including stability to low mode number magnetohydrodynamic (MHD) modes, energy confinement, helicity injection and current drive, the magnetic turbulence associated with this dynamo, and the beta (ratio of plasma and magnetic pressures) which can be supported in the geometry.
\end{abstract}

\section{INTRODUCTION}

The spheromak has been shown to be potentially a relatively simple fusion reactor with relatively low cost of electricity. ${ }^{1}$ The magnetic fields are generated primarily by plasma currents except for a vertical field, required to support the hoop stress, which is generated by a set of purely solenoidal coils. Current is driven by an external coaxial gun which generates linked toroidal and poloidal magnetic fluxes ("helicity") which drive current in the core of the spheromak through a magnetic dynamo. The current may return to the gun or be collected at the other end of the magnetic separatrix. It is anticipated that the ohmic heating from the plasma current will be sufficiently strong that no auxiliary heating source will be required to reach reactor conditions. ${ }^{2}$ We consider here physics issues which must be resolved to achieve the spheromak's promise.

\section{REACTOR CONFIGURATION}

Figure 1 shows an artist's conception of a spheromak reactor. The fusion plasma is confined within a magnetic separatrix with toroidal, magnetic flux surfaces; the geometry shown has two X-points. Helicity injection is from a coaxial, electrostatic gun shown located above the upper X-point; power flowing from the confinement region is diverted into the upper and lower coaxial regions which act as divertors. It may be possible to unbalance the magnetic configuration so that most of the energy and particles flow to the lower region, thus separating the divertor from the gun, and potentially improving the power and particle handling characteristics of the reactor.

The blanket, located in the annular region surrounding the plasma, is envisioned to consist of liquid lithium or lithium salts, perhaps flowing vertically between the wall and the plasma; solid or other configurations are also possible. If ohmic ignition occurs, the spheromak will allow a blanket without radial port penetrations. Perkins ${ }^{3}$ has suggested that the simplicity of the configuration may make a liquid/NaK potboiler reactor possible.

Energy confinement in the plasma will determine the optimum relationship among density, temperature, magnetic field, and plasma dimensions. However, deductions from low temperature experiments extrapolate to an extremely optimistic value at reactor conditions, and it is highly likely that different physics will determine the actual confinement. Accordingly, we use a simple estimate for thermal power production to provide approximate dimensions. Assuming electron and ion temperatures $T_{e}=T_{i}=20 \mathrm{keV}$ and equal deuterium and tritium densities, $n / 2$, expressed in terms of $\beta$ (the ratio of plasma and magnetic pressures), the deuterium-tritium power density, $P_{D T}$, at a magnetic field, $B$, is

$$
p_{D T}=1.14 \beta^{2} B^{4} \quad \mathrm{MW} / \mathrm{m}^{3} \text {. }
$$

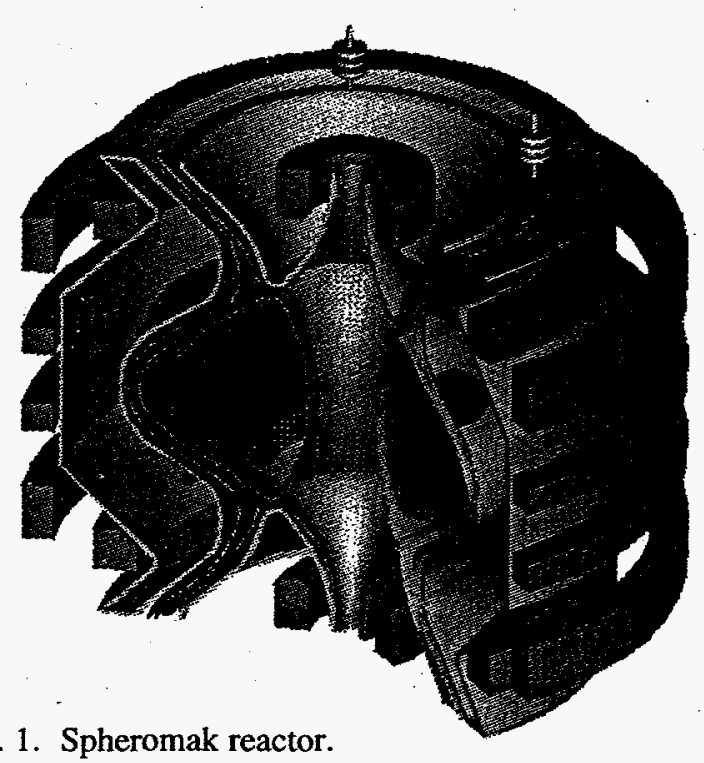

Fig. 1. Spheromak reactor. 
Taking a simple volume estimate $\mathrm{V}=\pi \kappa R^{3}$ with wall (flux conserver) radius $R$, and elongation $\kappa=1.5$, then gives the total fusion power production

$$
P_{D T}=5.4 \beta^{2} B^{4} R^{3} \quad \mathrm{MW} \text {. }
$$

Estimating $\beta=0.1$ (consistent with Sec. VI) and $40 \%$ conversion efficiency to electrical power, we require $B^{4} R^{3}$ $=4.6 \times 10^{4} \mathrm{~T}^{4} \mathrm{~m}^{3}$ for a $1 \mathrm{GWe}$ reactor.

The average neutron wall loading follows as

$$
p_{w}=0.065 P_{D T} / R^{2}
$$

Thus, for $1 \mathrm{GWe}$ and $p_{w}=8 \mathrm{MW} / \mathrm{m}^{2}$, we estimate $R=$ $4.5 \mathrm{~m}$ and $B=4.5 \mathrm{~T}$, so $n=1.4 \times 10^{20} \mathrm{~m}^{-3}$. The actual operating point will undoubtedly differ from this when the energy confinement and wall loading limits are better understood. Hagenson and Krakowski assumed a wall loading of more than twice our value and consequently found a more compact reactor.

\section{MAGNET AND PLASMA CONFIGURATION}

The hoop stress of the plasma current is supported by a solenoidal magnetic field which is curved to provide the detailed shape and elongation of the magnetic flux surfaces. Additional solenoidal coils are required in the coaxial gun to provide poloidal flux, as discussed in Section VII. Our reactor magnetic flux configuration is shown in Fig. 2. The zero flux surface, corresponding to the value on the geometric axis, is indicated by the heavy line. A conducting wall lies on this surface to slow the response of global plasma perturbations. On the resistive time of the wall, the equilibrium is supported by the external field. Thus, as in the tokamak, ${ }^{4}$ a vertical field of

$$
B_{v} \approx-\mu_{0} I L / 2 \pi R
$$

is required, where $I$ is the toroidal current in the spheromak and $L$ is a numerical factor of order unity. If the field were provided by a solenoid, it would require a current per unit length of $I L / 2 \pi R$. Thus, if discrete coils provide the vertical field, they will carry currents of order the plasma toroidal field. However, an external toroidal field, generated in the tokamak by a current greater by about $(R / a) q$, with $q$ the safety factor, is not required. As a result, it may be possible to construct the reactor using copper coils, although the detailed trade-offs of heating power and refrigerant, of capital investment, and of other factors, have not yet been examined carefully.

The actual shape of the separatrix is determined by the curvature of the external field. The shape also has a strong influence on the stability of the plasma to an $n=1$, MHD mode. Thus, if the vacuum field is curved away from the midplane, the plasma will become prolate. The prolate plasma is unstable to an internal tilt instability; in the approximation that the plasma current is modeled as a simple loop of radius $R / 2$, stability to tilt requires that the field index, $n_{i}$, satisfy

$$
n_{i}=-\left(\frac{R}{2 B_{v}}\right) \frac{\partial B_{v}}{\partial r}>1
$$

with $B_{v}$ the external field. This internal tilt mode is not strongly affected by the presence of a conducting wall, and so will grow on a fast time scale. Our configuration has been tested by the GATO code and shown to be stable against the internal tilt mode. ${ }^{6}$

If the field is mirror-like, with $n_{i}>0$, the plasma is unstable to a shift mode. However, this mode is stabilized by a conducting wall, and thus will grow only on the resistive time of the wall. Feedback control may be required on this much slower time scale.

The location of the X-points on the separatrix is determined by a balance between $B_{v}$, the poloidal field due to plasma current, and that associated with the coaxial gun. As noted below, this field also is closely related to the rate of helicity injection into the plasma, so that the determination of the field and currents is well specified, for example by the shape of the plasma boundary.

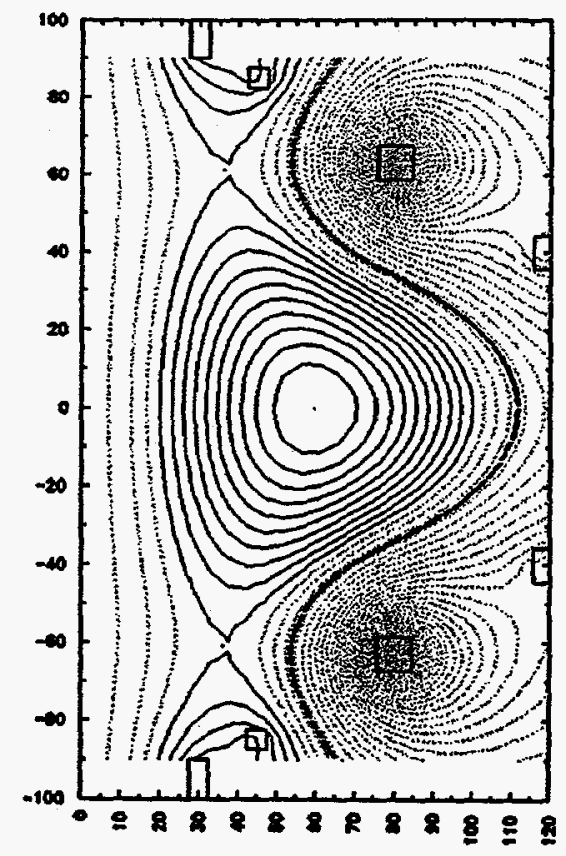

Fig. 2. Flux surface geometry. Helicity is injected by flowing current along the open flux surfaces from the upper to the lower divertor regions.

\section{STABILITY CONTROL; FEEDBACK SYSTEMS}

As noted in the previous section, the tilt/shift mode is stabilized by the conducting wall. On the resistive time scale of the wall, typically several 10 s of milliseconds, wall currents will decay and the tilt/shift mode is expected to become unstable. There may be plasma effects which will stabilize the mode; for example in the tokamak plasma rotation is known to be strongly stabilizing for similar modes. However, the conservative assumption given present knowledge is that a feedback system will be required. Thus eight feedback coils, four above and four below the midplane, are shown schematically in Fig. 1. 
Although a detailed analysis of feedback for a spheromak has not been made, results from other devices provide confidence that stabilization can be provided. The tilt/shift mode is the plasma equivalent of the instability of a current-carrying ring supported in a magnetic field. Feedback systems were used in levitrons to control the ring position to fractions of a millimeter ${ }^{7-9} \mathrm{MHD}$ modes have been stabilized in a tokamak ${ }^{10}$ and in a reversed-field pinch in the presence of a resistive wall, ${ }^{11}$ and the vertical $(m=0)$ mode is routinely stabilized in shaped tokamaks. Demonstrating feedback control, if needed, will be an important part of an advanced spheromak experiment.

\section{ENERGY CONFINEMENT}

The magnetic properties of spheromaks are confirmed experimentally, but confinement time. as reported in the literature has not appeared impressive, even taking into account the small size of experiments. Yet, by 1990, the CTX spheromak at the Los Alamos National Laboratory had produced $T_{e}$ up to $400 \mathrm{eV}$ with purely ohmic heating. ${ }^{12}$ In Ref. 2 , it is argued that these experimental results imply good confinement in the hot core of the plasma, comparable to early tokamaks and consistent with extrapolation to reactors such as the Hagenson and Krakowski design'. Ohmic heating alone may suffice to reach ignition in a spheromak, a great simplification in reactor design if true. Scenarios have also been developed ${ }^{13}$ for simultaneously building up the field by helicity injection and the temperature by ohmic heating to achieve ignition, with energy transport rates calibrated to CTX.

Briefly, the argument in Ref. 2 notes that understanding energy confinement requires focusing primarily on the confinement of the energy of the plasma (the usual meaning of $\tau_{E}$ ) and the energy stored in the magnetic field. These can be closely related, as discussed below. But because self-organization of the spheromak field continually regenerates field at the walls, any mechanism for destroying magnetic field near the wall is likely to dominate the magnetic field confinement time. This can mask good thermal energy confinement in the core if one examines only a global measure of confinement time determined from the magnetic decay time. Even in the optimum case, the low temperature of the plasma near the wall leads to much greater ohmic heating than in the plasma core. Nevertheless, all ohmic heating becomes irrelevant compared to alpha heating in the approach to ignition, so that the reactor power gain, $Q$, (fusion power divided by the power required to sustain the magnetic field) can be very large, about 100.1

Another consideration is the potential for excessive thermal heat loss due to magnetic fluctuations inherent in the self-organizing processes that maintain the magnetic field configuration. Magnetic turbulence may indeed have been the dominant cause for heat diffusion in the hot plasma core in CTX. However, it is argued $^{14}$ that magnetic turbulence diminishes as the electron temperature increases, to the point that other energy loss mechanisms, such as the electrostatic fluctuations thought to dominate energy confinement in tokamaks, become dominant in spheromaks at temperatures well below the ignition point (4 to $6 \mathrm{keV}$ ). Absent electrostatic losses, energy confinement at high temperatures in spheromaks would be much more favorable than was assumed in Refs. 1 and 13. The coupling of magnetic self-organization and thermal heat transport has been incorporated into a onedimensional transport model for spheromaks ${ }^{14}$, and this model, calibrated to CTX results, has been extrapolated to the reactor regime. ${ }^{15}$ Results reported in Ref. 15 support the conclusions above.

Briefly, the transport model has the form ${ }^{14}$

$$
\begin{gathered}
\frac{\partial}{\partial t} \frac{B^{2}}{2 \mu_{0}}+\frac{1}{r} \frac{\partial}{\partial r}(r P)=-\eta j^{2} \\
\frac{\partial}{\partial t} \frac{3}{2} n T+\frac{1}{r} \frac{\partial}{\partial r}\left(m \chi \frac{\partial T}{\partial r}\right)=\eta j^{2}
\end{gathered}
$$

where $\eta$ is the temperature-dependent plasma resistivity and $j$ is current density related to $B$ by Ampere's law. Coupling between these equations arises as both the Poynting vector $P$ and the thermal diffusivity $\chi$ are taken proportional to $\tilde{B}^{2}$, the mean-square magnetic fluctuation. $^{14}$ Moreover, through boundary conditions, $\tilde{B}^{2}$ can be eliminated, ${ }^{15}$ leading to a closed system of equations yielding the evolution of $B$ and $T$ in time. This step requires an additional assumption that forces $B(r)$ to assume a preferred fixed profile close to the relaxed, selforganized magnetic state found by Taylor, characteristic of spheromaks, reversed-field pinches, and various astrophysical systems. ${ }^{16}$

An open issue concerning this model is how the system can relax to a state of finite pressure even though the magnetic tearing modes that drive relaxation continue to be unstable if the pressure is finite. The resolution of this issue appears to be related to the finite rate of heat transport due to magnetic turbulence. Then the relaxed state would not be a stable, Taylor state, but rather a state of minimal transport, the actual state of finite pressure being a balance between power sources and this minimal transport at that pressure. ${ }^{17}$

\section{BETA LIMITS}

Previous calculations of the beta limits in spheromaks ${ }^{18-22}$ have been extended to a geometry with a separatrix which couples to a plasma gun or other external helicity injector. Experiments often reached higher beta than predicted from the Mercier limit with expected current profiles. ${ }^{23}$ The reasons are not understood; we assume that the Mercier limit is the pertinent condition, so that the shape of the magnetic shear is the important variable in determining beta.

We examined the effect of the current profile, $\mathbf{j}$, (and thus shear) by parameterizing the current profile as 
$\lambda(\psi) \equiv \frac{\mu_{0} \mathbf{j} \cdot \mathbf{B}}{B^{2}}=$ const $*\left(1+a_{1} \psi+a_{2} \psi^{2}+a_{3} \psi^{3}+\cdots\right)$

where $\psi$ is the poloidal flux. The constant is determined by the equilibrium calculation. The case $\lambda=$ const. with $\beta=0$ is the Taylor equilibrium. To first approximation, the shear is proportional to the difference between the safety factor on axis and that on the edge of the confinement volume. The safety factor on the magnetic axis can easily be shown to be

$$
q_{0}=2 / \lambda_{0} R_{0}
$$

where $R_{0}$ is the radius at the magnetic axis; the flux surface has been assumed circular in the limit of small minor radius. We characterize the maximum beta by $\beta_{p}$ defined as the volume-averaged pressure divided by the surface-averaged, squared poloidal magnetic field:

$$
\beta_{p} \equiv 2 \mu_{0}\langle p\rangle_{v o l} /\left\langle B_{p}^{2}\right\rangle_{\text {edge }}
$$

For the case in which there is a flux hole without current through it, so that the edge $q$ is 0 , we will find that the maximum Mercier-stable $\beta_{p}$ is proportional to $q_{0}$. The cases with current through the hole will be seen independent of $q_{0}$.

We require $\lambda$ to vary monotonically from the magnetic axis to the edge; calculations show that the maximum $\beta_{p}$ occurs when $d \lambda d \psi=0$ on the axis and edge. The result of varying $q_{0}$ without current in the flux hole along the geometric axis, thus with no external magnetic field, is presented in Fig. 3. Clearly, the achievable beta is dependent on the current profile for this case.

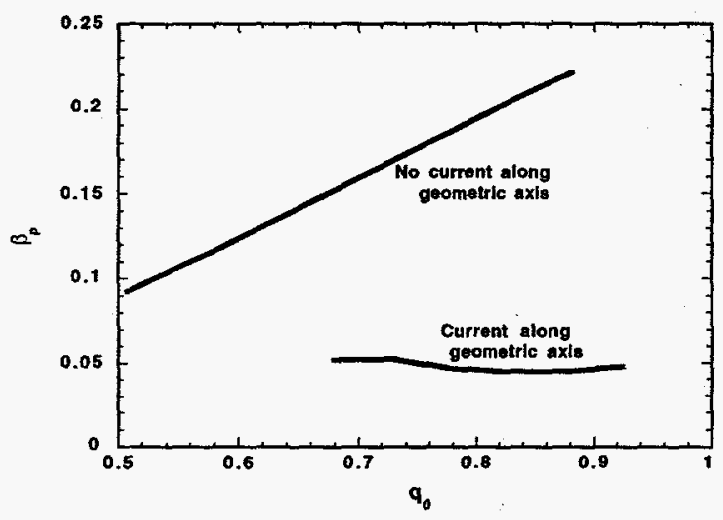

Fig. 3. Maximum Mercier-stable $\beta_{p}$ as a function of $q_{0}$ with and without current along the geometric axis.

If there is current along the geometric axis, and thus a toroidal magnetic field on the separatrix, the safety factor will diverge there. The effect of this divergence is to generate a large shear on the plasma edge while reducing the shear in the volume. For the particular geometry of Fig. 2, with $\lambda=$ const. outside the separatrix, the result is to reduce the maximum beta and make it independent of $q_{0}$. The quantitative result is now dependent on the details of the flux-hole size as well as where external currents flow; clearly, further study of the beta-limit for this case is needed. Alternatively, if helicity injection can be achieved with no currents along the geometric axis, the beta can be maximized at a level consistent with the requirements of constructing a reactor of minimum size.

\section{CURRENT DRIVE AND THE PLASMA GUN}

The spheromak configuration is close to a minimum energy state subject to the constraint of the conservation of helicity, ${ }^{16} \mathrm{~K}$, a measure of linked poloidal and toroidal magnetic fluxes. On the resistive time scale, helicity decays and must be replenished to sustain the configuration. The plasma gun is a means of injecting the helicity into the flux-conserving wall by means of a voltage, $V_{g}$, across a poloidal flux entering the volume from the gun, $\psi_{g}$, yielding a balance ${ }^{24}$

$$
\frac{d K}{d t}=2\left(V_{g} \psi_{g}-\int \eta \mathbf{j} \cdot \mathbf{B} d V\right)
$$

where the integral is over the volume. In the approximation that $\lambda$ is constant on the open flux, the injected current is $I_{i n j}=\lambda \psi_{g} / \mu_{0}$, yielding a gun impedance of $\int \eta B^{2} d V / \psi_{g}^{2}$.

We estimate the gun parameters from the power injected, $V_{g} I_{g}$, which balances the net power losses, $P_{D I} l Q$. Consequently, for $Q=100$ the injected power is $1 \%$ of the fusion power; about $250 \mathrm{MW}$ in the present case. The magnetic field of $5 \mathrm{~T}$ with $R \approx 3 \mathrm{~m}$ implies a plasma current of about $I_{p}=60 \mathrm{MA}$; an amplification factor $\left(l_{p} / I_{g}\right)$ of 60 will require a gun current of $1 \mathrm{MA}$ at a voltage of $250 \mathrm{~V}$. Estimating $\lambda=5 / R$, the gun flux is $\sim 0.6$ Weber, requiring a area of 0.1-0.2 $\mathrm{m}^{2}$ and, at a circumference of $2 \pi R$, a flux bundle width of a few $\mathrm{cm}$. The open flux is thus small compared with the closed flux ( $\sim 30$ Weber), as required for the edge ohmic power losses to be small.

\section{DIVERTOR}

The magnetic geometry shown in Figs. 1 and 2 has a natural divertor. Although the poloidal configuration is similar to that of a tokamak, the distance along a field line to the divertor region is shorter because of the lack of external toroidal field. On the other hand, the system is effectively open as there is no interference from toroidal field coils, so that the plasma losses can be spread over as large an area as needed to handle power losses.

The fusion alpha particle power in a 1 MWe plant is about $500 \mathrm{MW}$; consequently, the power carried in the scrape-off layer is three times that injected by the gun. The edge plasma will thus be relatively hot, reducing the ohmic losses from the gun current. An increase in electron temperature from $50 \mathrm{eV}$ to $200 \mathrm{eV}$ decreases the ohmic edge losses by a factor of 8 , so the additional heating is beneficial to the efficiency of helicity injection. 
Experiments will be required on injecting helicity in the presence of the plasma losses, but the effects of the fusion power should be positive for reactor operation.

\section{BLANKET}

The blanket in a spheromak reactor could be liquid lithium or liquid salts, that serve all the functions of a blanket in other fusion concepts (heat transfer, breeding) and shield the reactor vessel from severe neutron damage. Heat transfer by a flowing liquid wall that absorbs most of the neutron energy may also make possible much higher power density on the wall than would a conventional metal or ceramic wall dependent on coolants flowing through structures in and behind the wall. This duel possibility of neutron protection and high power density motivated the application of a liquid blanket for fusion reactors, first for the Astron magnetic concept, ${ }^{25}$ and more recently for the HYLIFE inertial fusion design. ${ }^{26}$ Protection from neutrons extends the life of reactor structures and reduces the fluence they receive, in some design studies to the point that components last for the life of the plant and allow "shallow burial" upon decommissioning according to present U.S. regulations. ${ }^{27}$ The longer lifetime of components, together with higher power density, could significantly reduce the cost of electricity from fusion power plants. ${ }^{28}$

Several technical problems must be addressed to utilize liquid blankets in a spheromak. ${ }^{29}$ (1) The flowing liquid must act like a conducting wall for the tilt/shift instability. (2) Evaporated liquid must be efficiently ionized and transported away (along field lines) to prevent penetration into the burning plasma. (3) Access must be available for entry and exit of the liquid and flow paths established that leave ample space for the plasma inside the protective liquid mantle. (4) The liquid medium must be capable of efficient heat transfer and adequate breeding of tritium. (5) The liquid blanket must not contain materials that exacerbate environmental and safety concerns. A liquid wall composed of non-flammable Flibe molten salt appears suitable for this. ${ }^{29}$

\section{ACKNOWLEDGMENTS}

We particularly want to thank L. Donald Pearlstein for his help, including providing the means of calculating the spheromak equilibrium and stability. This work was performed under the auspices of the U.S. Department of Energy by LLNL under contract W-7405-ENG-48.

1. R. L. Hagenson and R. A. Krakowski, Fusion Techn. 8, 1606 (1985).

2. T. K. Fowler, J. S. Hardwick, and T. R. Jarboe, Comments Plasma Phys. Contr. Fusion 16, 91 (1994).
3. L. J. Perkins, private communication (1995).

4. John Wesson, Tokamaks (Oxford Science Pub., Clarendon, Oxford 1987), Sec. 3.7.

5. S. C. Jardin, M. S. Chance, R. L. Dewar, R. C. Grimm, and D. A. Monticello, Nucl. Fusion 21, 1203 (1981).

6. Alan Turnbull, private communication (1996).

7. O. A. Anderson, et al., Plasma Physics and Controlled Nuclear Fusion Research 1971, (IAEA, Vienna, 1971), Vol I, p. 103.

8 . R. Freeman, et al., op cit., Vol I., p. 27.

9. M. W. Alcock, et al., Plasma Physics and Controlled Nuclear Fusion Research 1974, (IAEA, Vienna, 1974), Vol II, p. 77.

10. A. W. Morris, et al., Phys. Rev. Letters 64, 1254 (1990).

11. B. Alper, Phys. Fluids B 2, 1338 (1990).

12. T.R. Jarboe, F.J. Wysocki, J.C. Fernández, I. Henins, and G. J. Marklin, Phys. Fluids B 2, 1342 (1990).

13.J. S. Hardwick, Ohmic Ignition in a Spheromak, $\mathrm{PhD}$ Dissertation, Dept. of Nucl. Eng., Univ. of Cal., Berkeley, 1995.

14. T. K. Fowler, Fusion Techn. 29, 206 (1996).

15. T. K. Fowler and D. D. Hua, J. Fusion Energy 14, 181 (1995).

16. J. B. Taylor, Rev. Mod. Phys. 58, 741 (1986); Phys. Rev. Letters 33, 1139 (1974).

17. T. R. Jarboe, Plasma Phys. Control. Fusion 36, 945 (1994).

18. M. Okabayashi and A. M. M. Todd, Nucl. Fusion 20, 571 (1980).

19.P. Gaiter, R. Gruber, and F. Troyon, Nucl. Fusion 21, 1399 (1981).

20. S. C. Jardin, Nucl. Fusion 22, 629 (1982).

21. R. M. Mayo and G. J. Marklin, Phys. Fluids 31, 1812 (1988).

22. G. Marklin, Proc. US-Japan Workshop Field-Reversed Config. with Steady-State High-Temp. Fusion Plas. and 11 th US-Japan Workshop Comp. Toroids, Nov. 1989, LANL report LA-11808-C, p 181, May 1990.

23. F. J. Wysocki, J. C. Fernández, I. Henins, T. R. Jarboe, and G. J. Marklin, Phys. Rev. Letters 61, 2457 (1988).

24. J. B. Taylor and M. F. Turner, Nucl. Fusion 29, 219 (1989).

25. N. C. Christofilos, "Design for a High Power Density Astron Reactor," LLNL Report UCRL-72957 (1971); J. Fusion Energy 8, 97 (1989).

26. R. W. Moir, R. L. Bieri, and X. M. Chen, Fusion Techn. 25, 5 (1994).

27. J. S. Latkowski, Inertial Fusion: A Clearer View of the Environmental and Safety Perspectives, $\mathrm{PhD}$ Dis., Dept. of Nucl. Eng., Univ. of Cal., Berkeley, 1996.

28. R. W. Moir, Nuclear Eng. Design 29, 34 (1995).

29. R. W. Moir, "Liquid First Walls for Magnetic Fusion Energy," LLNL Report UCRL-ID-123902, March 28, 1996. 